\title{
Managing Mutilating Hand Injuries
}

Amitabha Lahiri, MBBS, MS, MCh (Plast), FRCS (Edin), FAMS (Hand Surgery), MRes (tissue engineering)

\section{KEYWORDS}

- Hand • Trauma • Reconstruction • Microsurgery • Rehabilitation

\section{KEY POINTS}

- Management of mutilating injuries is complex and should be based on clear understanding of principles of wound management, fracture fixation, and soft-tissue reconstruction.

- The surgeon must be able to anticipate the effects of the trauma and set clear goals of reconstruction directly from the first surgery.

- Debridement, bony stabilization, revascularization, and soft-tissue cover should be achieved in the primary surgery. Nerve and tendon reconstruction can be performed as delayed procedures.

\section{INTRODUCTION}

The hand is a complex organ that is unique in several ways. It has a mechanical component consisting of bones, joints, tendons, and muscles, and a sensory component comprising the skin and the sensory nerves. The components work seamlessly to give rise to the function of the hand, which is referred to as prehension.

Prehension includes the integration of 2 components: feeling, which is transfer of information from the environment to the brain, and response, the manipulation of objects.

Both the components are of equal importance. Loss or damage to any component in the system results in suboptimal function. An insensate hand in leprosy is as dysfunctional as a sensate but paralyzed hand in poliomyelitis.

"Mutilating hand injuries" is a group of injuries that is difficult to define. The English dictionary defines mutilate as "to damage something severely, especially by violently removing a part."

Mutilation of the hand may be taken to include injuries that result in significant damage to several tissues at once.

The patterns of these injuries are so variable because of the nearly unlimited permutations and combinations of the severity, extent, and the number of tissues involved that the treatment can never be standardized; however, clear principles for surgical management can be established.

The surgeon managing such injuries must have a clear understanding of the principles that would influence decisions and hence the outcomes. A clear treatment plan based on precise goals leads to rapid functional and psychological recovery. However, repeated surgeries and failures often may lead to opposite outcomes.

The ability to restore function in the injured hand nowadays is the result of the culmination of developments in several fields.

- Principles of debridement

- Antibiotic therapy and infection

- Principles and techniques of skeletal stabilization

- Principles and techniques of tendon repair

- Microvascular surgery

- Peripheral nerve surgery

- Tissue transfer

- Rehabilitation

Thus, a surgeon or a team dealing with mutilating hand injuries must be conversant with these principles in order to provide the optimal management.

For the purpose of the present discussion, pure amputation injuries are excluded; however,

The author has nothing to disclose.

Department of Hand and Reconstructive Microsurgery, National University Hospital, Level 11, NUHS Tower block, 1E Kent Ridge Road, Singapore 119228, Singapore

E-mail address: amitabha27r@gmail.com 
amputation in the context of a mutilating injury is discussed.

\section{THE INJURY}

In the current day and age, such an injury usually results from heavy machinery or automobile accidents. It may also arise from explosives in war zones or from the hand being trapped under heavy rubble in natural disasters.

The common characteristics of mutilating injuries can be summarized as follows.

- Loss of skin and soft-tissue cover

- Multiple tendon injuries with loss of tendon substance

- Wide zone of injury to the vessels with devascularization of parts

- Nerve injuries with loss

- Fractures and dislocations

- Contamination of tissues with foreign material

\section{DEFINING THE GOALS OF SURGICAL MANAGEMENT}

The final functional outcome of each injury is unique and varies with the severity of structural damage and contamination, as well as the surgery and rehabilitative care; however, some generalization can be established.

The overall goal of reconstruction is summarized as:

- Restoration of maximal function

- In shortest possible time

- Through minimum number of procedures

- If multiple procedures are planned, each procedure should be performed with clear goals and should set the stage for the next procedure

\section{DEFINING THE FUNCTIONAL HAND}

As already mentioned, the function of the hand is summarized by the word "prehension" and is a composite function of dynamic interaction between the motor and sensory loops that connect the hand and the nervous system.

The motor function relies on the integrity of the motor nerves, muscles, tendons, bones, and joints. Sensory function relies on the integrity of skin cover, receptors, and the sensory nerves.

In 1979 Midgley and Entin ${ }^{1}$ summarized the 6 basic requirements in a functioning hand:

- Strength

- Position

- Length

- Stability

- Mobility

- Sensibility

\section{FUNCTIONAL ASPECTS OF THE HAND}

Essentially the functional hand depends on an opposable thumb and one or more mobile digits. ${ }^{2}$

The opposable thumb provides a post against which the digits grip objects.

A pinch can be achieved with the thumb and a single mobile digit.

A tripod pinch requires the thumb and 2 digits (Figs. 1 and 2).

The power grip requires 3 to 4 digits and the thumb.

Span requires ulnar digits.

The ability of the hand to adapt. The surgeon must remember that the hand shows extraordinary
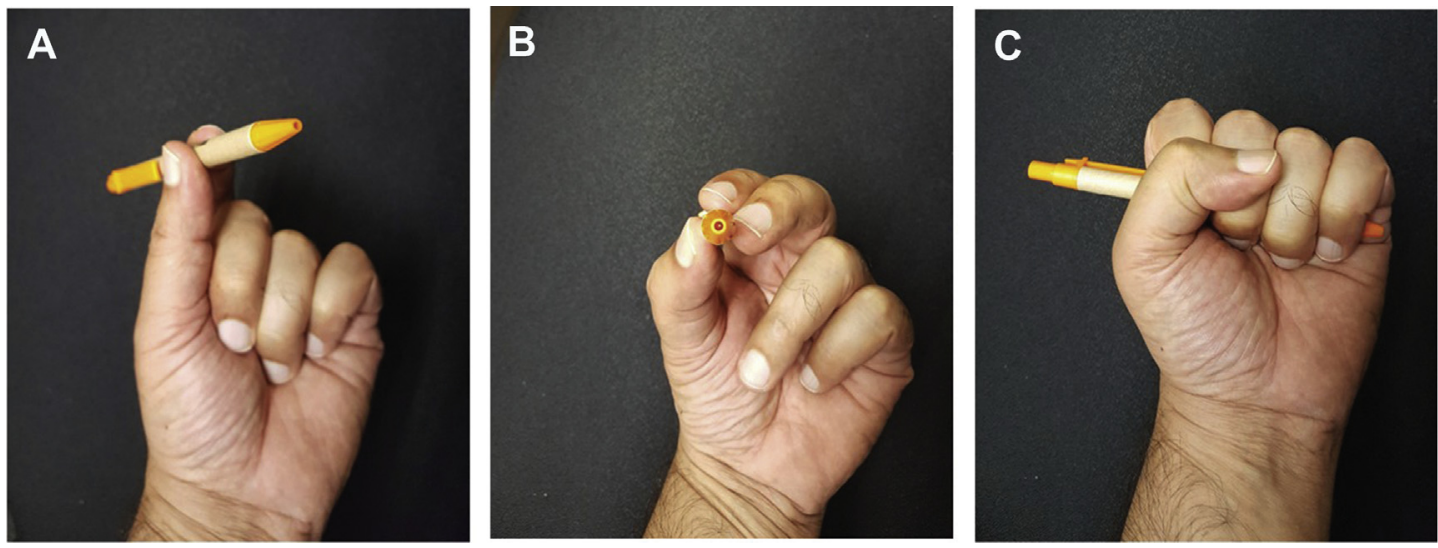

Fig. 1. (A) Pinch can be achieved using one finger and the thumb; however, it lacks strength and the ability to stabilize the object being held. $(B)$ A tripod pinch requires 2 fingers and the thumb. It has precision strength and control, but large objects cannot be held using this grip. $(C)$ Power grip using all the fingers and the thumb demonstrates strength and stability. 

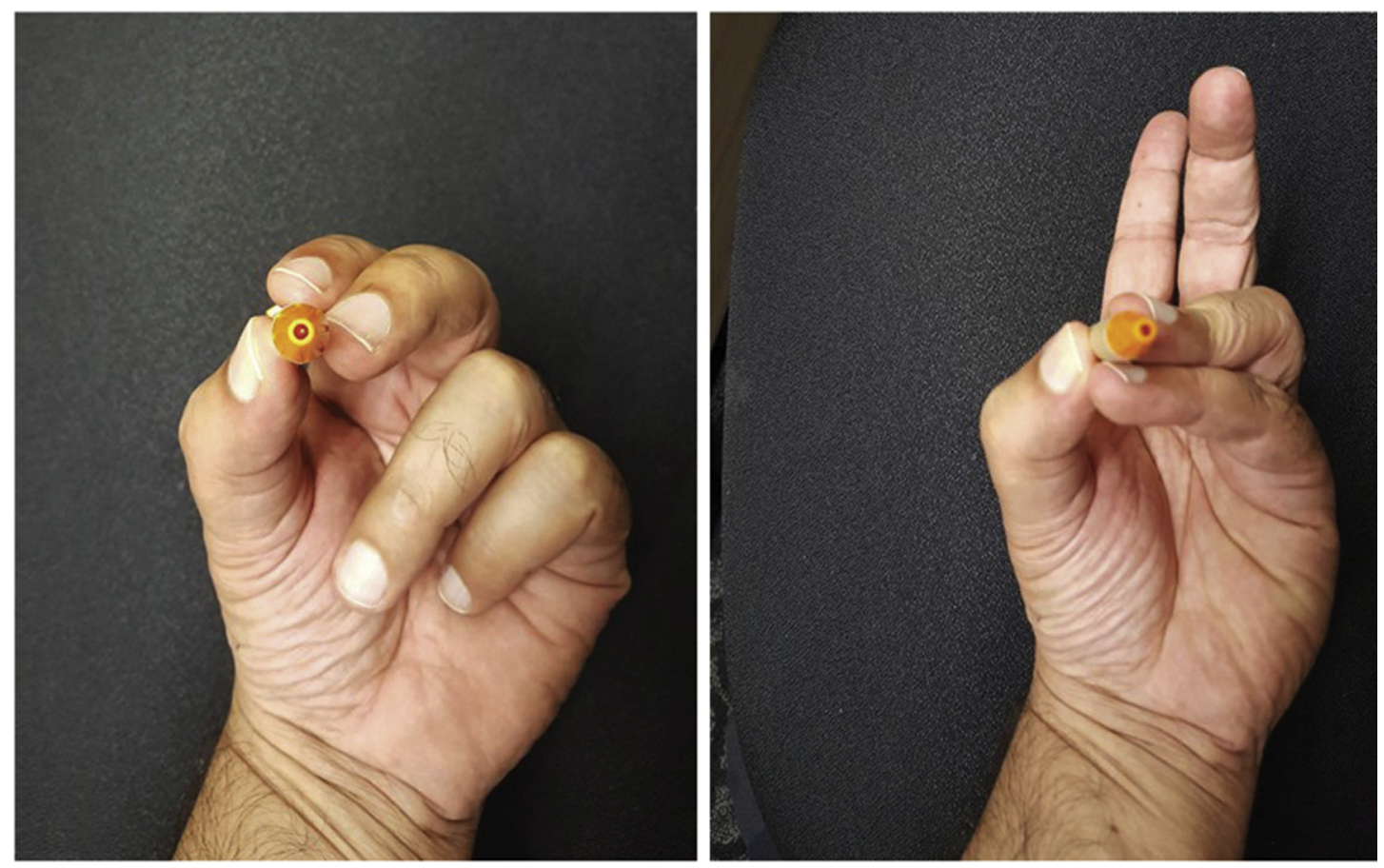

Fig. 2. The ability for the hand to adapt and compensate for the loss of digits. A tripod pinch can be achieved with the radial or ulnar digits against an opposable thumb.

plasticity. In situations of significant structural loss, the remaining functional elements can adapt to compensate for the lost function.

In general the functions involving precision are performed by the radial 2 digits and the thumb, whereas the functions for holding large objects are performed by the entire hand and needs the ulnar digits and the thumb. The thumb plays the crucial part in both forms, although the digits can adapt to the function.

The implication of this concept is that fewer but functional digits, whether radial or ulnar, may provide equal or better outcomes than a greater number of stiff or nonfunctional digits.

\section{APPROACH TO A PATIENT WITH A MUTILATING INJURY OF THE HAND}

The management starts with a precise clinical assessment of the patient followed by the creation of a surgical plan.

Clinical assessment. The most essential part of clinical assessment of a patient with major hand trauma is the assessment of the patient as a whole, and exclusion of severe life-threatening trauma and exsanguination (Fig. 3).

It is also essential to examine the whole upper limb including the axilla, shoulder, and clavicular region in such cases.
Once the patient is stabilized, attention is focused on the hand.

A history of comorbidities and possible drug allergies that may compromise the safety of major surgery is extremely important.

Three essential questions need to be asked regarding the trauma to the hand.

1. When: the time at which injury took place

2. How: the mechanism

3. Where: the environment in which the injury occurred (possible contaminants)

Most of the time the patient is in severe pain and in a state of psychological shock, at which point a conventional examination may not be feasible; however, a detailed knowledge of the structural damage is essential in formulating the surgical strategy.

Careful observation and synthesis of information is gained from:

- Knowledge of surface anatomy

- A visual "feel" for the depth of trauma

- Understanding the mechanism of injury

- Plain radiographs

These factors provide an extraordinary amount of information without the need to touch the patient. 


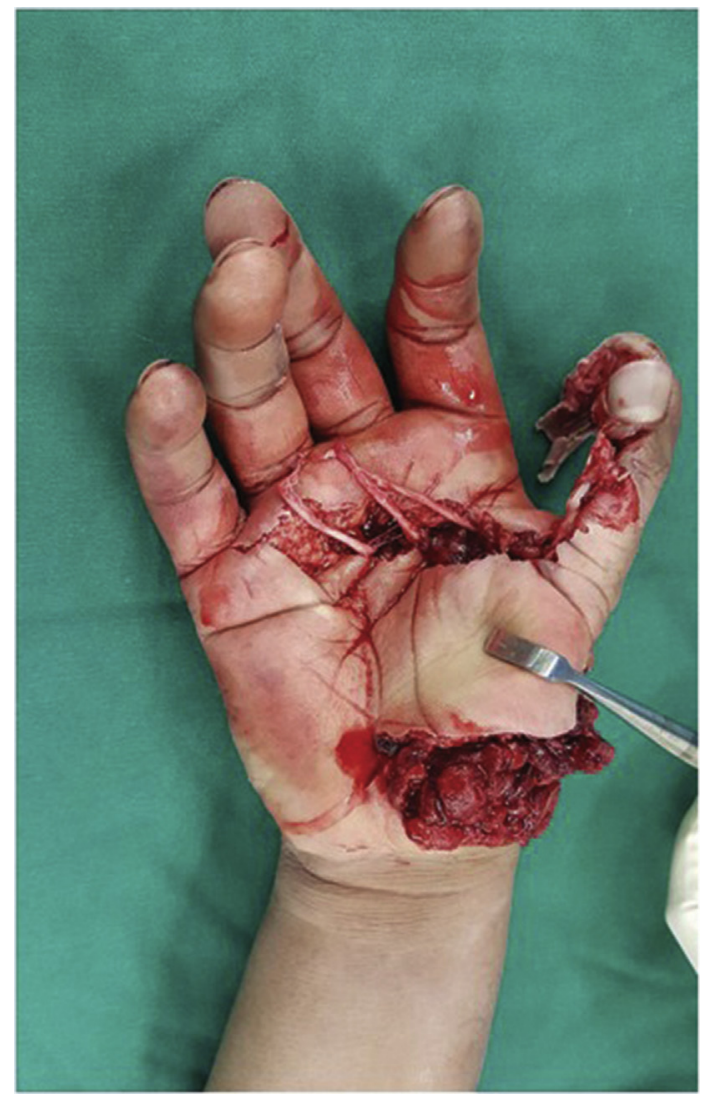

Fig. 3. A hand crushed by heavy machinery. Conventional examination is practically impossible in the awake patient; however, a thorough visual examination can provide a great amount of information that is necessary for planning. On visual assessment, the thumb shows separation of soft tissue from bone and the distal soft tissue is rotated, indicating likely devascularization of distal phalanx. The thenar muscles are crushed and seen protruding and are likely to be unsalvageable, while the thenar skin is likely to be devascularized because of separation from muscles. Avulsed digital nerves are seen, indicating a high possibility of neurovascular trauma even though the color of the digits is pink. The middle finger is rotated, indicating a possible fracture.

The wound may be irrigated with saline in the emergency department and photographs taken so that repeated examinations by different specialists are avoided.

\section{PLANNING}

Table 1 and a sketch can enhance preoperative planning.

Surgical preparation. It is useful to refer to the plan for surgical preparation. Anticipation of potential donor sites must be included in the patient's preparation on the table. Failure to anticipate may require interruption of surgery to prepare additional sites for donor tissues.

Common examples are:

Skin graft: thighs

Pedicled flaps: abdominal flap/groin flap

Free/flow-through flaps: anterolateral thigh flap, dorsalis pedis, radial artery forearm flap, and lateral arm flap from the opposite limb

Vein graft: leg (saphenous vein)

Nerve graft: leg (sural nerve)

Spare parts. Considering spare parts is one of the essential components of surgery, which reduces the burden on healthy donor sites in the body. ${ }^{3}$

Tissues that are not crushed or contaminated but are being sacrificed can be used as spare parts.

For example, a digit with double-level injury that is not being replanted can be a source of nerve graft, artery, and skin.

Free flaps can be fashioned from amputated parts that are not being replanted.

Degloved or sheared skin can be used as a skin graft after removal of fat.

\section{STEPS OF SURGERY}

Debridement. Debridement is the first and essential component of surgery, and is performed in a methodical fashion to include skin, subcutaneous tissue, muscle, and bone while preserving nerves and intact vessels and tendons.

The debridement should create unquestionably healthy surgical margins for each tissue. A temptation to preserve devitalized tissue should be avoided.

A good debridement sets the stage for clear reconstruction goals and prevents postoperative complications such as ongoing tissue necrosis and infection that require repeated debridement.

Intraoperative "triaging" and the sequence of salvage and prioritization (thumb). In mangling injuries the different structures suffer different degrees of damage. The surgeon must make decisions on the spot regarding the order in which the structures are to be addressed to achieve the best outcomes. ${ }^{4}$

Fatigue from spending several frustrating hours on salvaging a severely damaged digit may compromise the reconstruction of the better preserved digits and worsen the surgical outcome in comparison with successfully salvaging the less severely damaged digits and sacrificing an unsalvageable digit.

However, the decision to sacrifice a digit is not simple. A digit may be deemed unsalvageable if 


\begin{tabular}{|c|c|c|c|}
\hline$\underline{\text { Structures }}$ & Extent of Injury & Plan & Donor \\
\hline \multicolumn{4}{|c|}{ Immediate priority for repair/reconstruction } \\
\hline Dorsal and palmar skin & Area of loss & Flap/graft & Thigh/groin flap \\
\hline $\begin{array}{l}\text { Palmar arch and digital } \\
\text { arteries }\end{array}$ & $\begin{array}{l}\text { What level/possible } \\
\text { segmental loss }\end{array}$ & Vein graft & $\begin{array}{l}\text { Foot/leg/forearm/ } \\
\text { flow-through flap }\end{array}$ \\
\hline Fractures & $\begin{array}{l}\text { Level/bone loss/ } \\
\text { comminution }\end{array}$ & $\begin{array}{l}\text { Plating/bridge plating } \\
\text { external fixation/bone } \\
\text { graft? }\end{array}$ & Donor? \\
\hline \multicolumn{4}{|c|}{ Possible secondary reconstruction } \\
\hline $\begin{array}{l}\text { Median/ulnar and } \\
\text { digital nerves }\end{array}$ & $\begin{array}{l}\text { Level of trauma/possible } \\
\text { segmental loss }\end{array}$ & Primary repair/graft & Forearm/sural \\
\hline $\begin{array}{l}\text { Flexor and extensor } \\
\text { tendons }\end{array}$ & $\begin{array}{l}\text { Which level and digit } \\
\text { Possible segmental loss }\end{array}$ & $\begin{array}{l}\text { Primary repair/delayed } \\
\text { grafting }\end{array}$ & \\
\hline
\end{tabular}

the anticipated outcome is a short or a nonfunctional digit.

1. The skin and neurovascular bundles are crushed or avulsed over a significant length

2. Large segments or multiple phalanges have bone loss or loss of multiple joints

3. Severe contamination of tissues

Priority is given to salvaging the thumb. However, if the thumb is not salvageable, reconstruction must set the stage for further reconstruction.

Midgley and Entin's ${ }^{1}$ parameters of strength, position, length, stability, mobility, and sensibility can be used here to anticipate the functional outcome of the digits.

In summary, following adequate debridement,

1. Reconstruction should begin with salvage of best-preserved digits (author's opinion)

2. High priority must be given to salvage the thumb

3. If the thumb or several digits are not salvageable, using another amputated digit may be considered to reconstruct the thumb if possible (transposition). If transposition is not thought to be suitable, flap cover for secondary reconstruction of the thumb (osteoplastic reconstruction), or a toe transfer, may be incorporated in the procedure. Essentially the end point of the first procedure should prepare the stage for a secondary reconstruction.

\section{Essential Components of Primary Surgery}

The primary surgery has three essential components.
Skeletal stabilization. Skeletal stability forms the foundation for soft-tissue reconstruction.

The aim is to maintain length and alignment of the skeletal elements. In many cases shortening of bones can provide a platform for primary cooptation of neurovascular structures and assist in achieving soft-tissue cover. However, excessive shortening of skeletal elements may deform the hand and compromise function.

The options include internal fixation with plates and screws or Kirschner wires.

In situations of bone loss, external fixators or bridging plates are used.

When using external fixators, it must be kept in mind that positioning of the hand for microvascular procedures may become extremely difficult and, similarly, application of groin or abdominal flaps may be impossible.

The choice of fixation is also dictated by the contamination and the anticipated risk of infection during and following the debridement. The assessment is entirely subjective; however, in situations of biological contamination (e.g., barnyard injuries, contamination with meat or fish), external fixation may be preferred because of the higher risk of deep infection, which may necessitate removal of implants.

Revascularization. Following skeletal stabilization, the immediate concern is to establish blood flow to the ischemic structures. One or several digits may be devascularized, and crush injuries resulting in mutilation commonly result in loss of large segments of blood vessels, meaning that vein grafts are necessary.

Vein grafts can be harvested from the same limb if the proximal forearm is not injured. Saphenous vein may also be used. 
Although vein grafts are possible for veins they have a higher propensity to fail, and in this situation the veins should not be reversed. Once revascularization has been performed, nerves and tendons can be repaired at the same sitting or planned for a secondary reconstruction (Fig. 4).

Soft-tissue cover. ${ }^{5}$ The next crucial component of primary reconstructive surgery is soft-tissue cover. Bones, implants, or vessels cannot be left exposed. If these structures are covered, the remaining soft-tissue defect can be reconstructed as an early secondary reconstruction within 48 to 72 hours.

Pedicled groin and abdominal flaps alone or in combination provide a versatile solution for emergency flap coverage of the hand. These flaps can be raised rapidly to cover a variety of defects and may be combined to provide coverage for large areas. ${ }^{6}$

Anterolateral thigh flaps can provide reliable soft-tissue cover for the injured hand. They can also be used as flow-through flaps to provide simultaneous revascularization. ${ }^{7}$

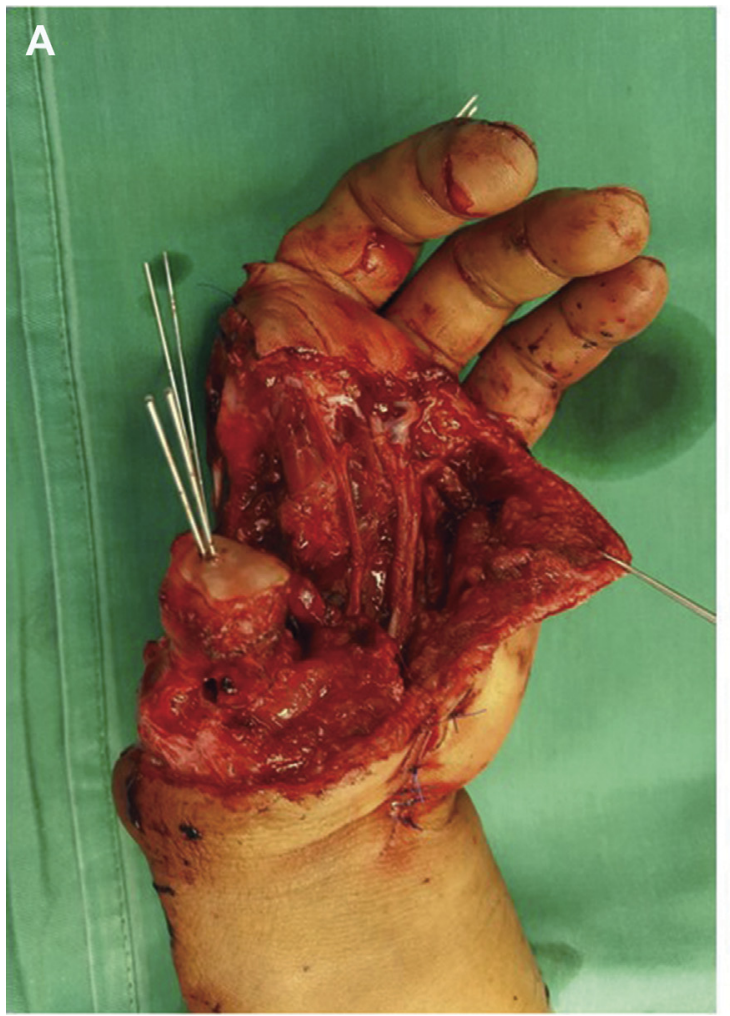

Free flaps also provide an opportunity for singlestage reconstruction of composite soft-tissue defects of the hand. A dorsalis pedis flap can be harvested with extensor tendons ${ }^{8}$ to reconstruct dorsal defects with loss of tendons. Similarly, radial forearm flaps and lateral arm flap can be harvested with vascularized bone to treat bone and soft-tissue defects (Fig. 5).

\section{Late Secondary Procedures}

The primary procedure should set the stage for necessary secondary procedures. ${ }^{9}$

Secondary procedures should be carried out when the wounds have healed and the scars have stabilized. Such procedures include bone grafts to bridge bone defects, tendon grafts, tendon transfers, nerve grafts, and toe transfer.

\section{HINDRANCES TO IDEAL OUTCOME AND THE ROLE OF REHABILITATION}

Beyond soft-tissue cover and survival of digits, there are several barriers to establishment of function.

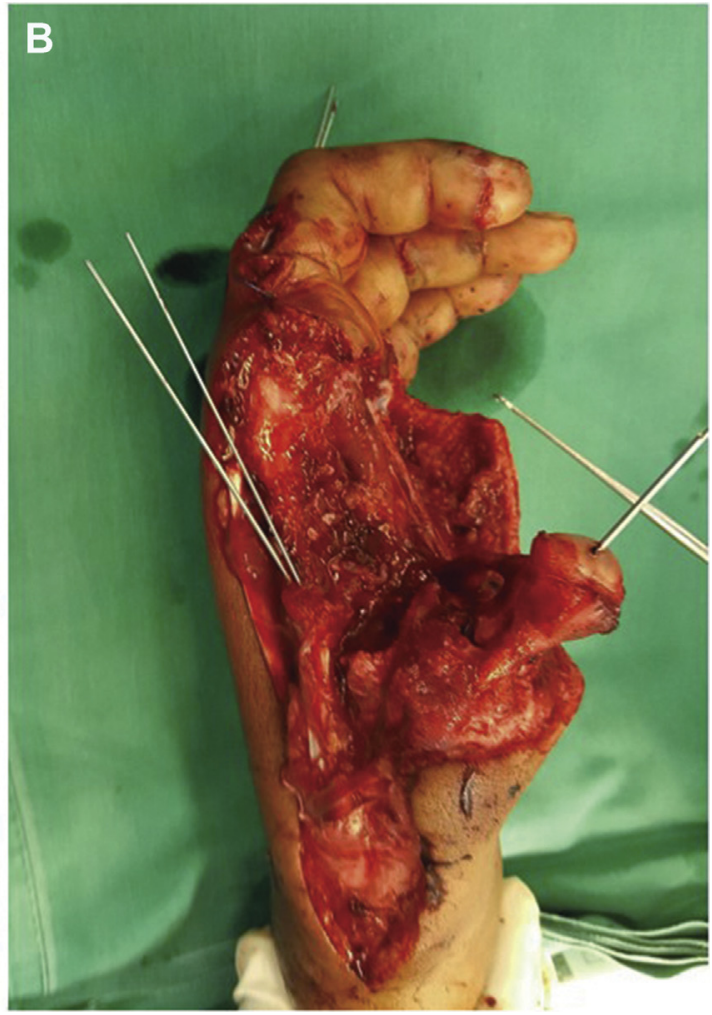

Fig. 4. Complete debridement of crushed tissues. Following revascularization of digits using vein grafts, the index finger could not be perfused. Exploration showed thrombosis of both digital arteries up to the distal phalanx. The decision was made to amputate. $(A)$ The thumb metacarpal, completely devoid of soft-tissue cover, was preserved. $(B)$ Skeletal stabilization of the thumb metacarpal to the trapezium in the position of function, and stabilization of the third carpometacarpal joint. 


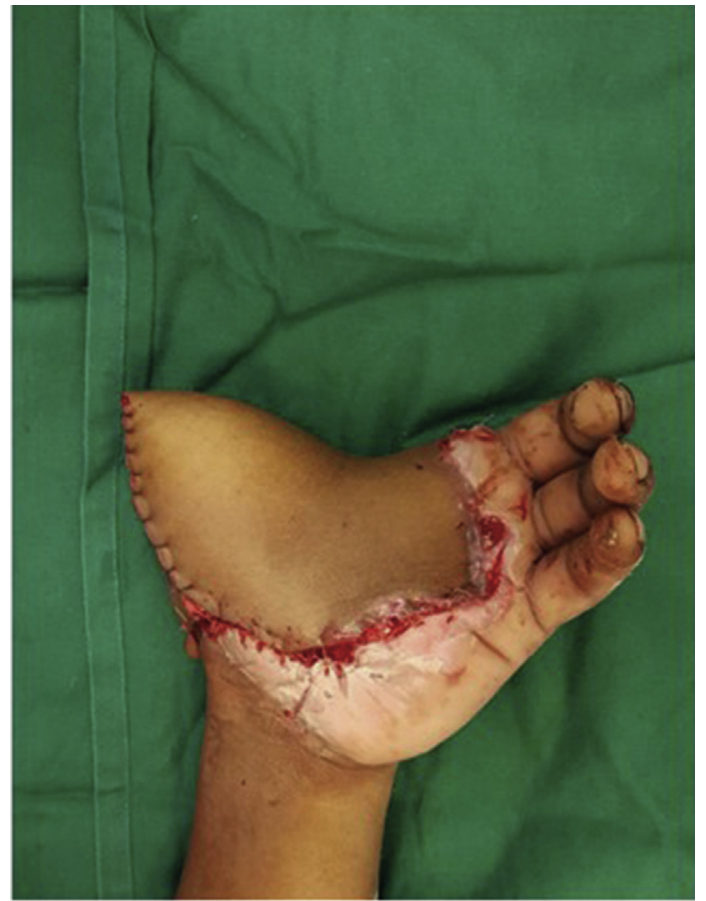

Fig. 5. Combined groin and abdominal flap transfer was performed during the primary surgery to reconstruct the palm, the thumb, and the dorsum. The soft-tissue cover over the thumb metacarpal was planned for secondary debulking, and also provided a platform for a possible great toe transfer.

Mutilating injuries involve multiple tissue planes that heal by fibrosis. Adhesions and loss of gliding planes through scarring impair tendon function and joint motion. Nerve repairs in general have partial recovery, which is caused by axonal entrapment at the site of repair and loss of axons caused by fascicular mismatch.

The surgeon must anticipate individual problems and initiate rehabilitative measures as early as possible.

The rehabilitation in such cases is complex, and set protocols cannot be applied. Each patient requires an individualized protocol. For example, combined flexor and extensor injuries in different digits and at different anatomic zones along with fractures would produce unique scenarios for each patient. There has to be clear communication between the surgeon and the occupational therapist in formulating a plan that is safe to ensure maximal functional recovery.

\section{THE FUTURE: BIONIC PROSTHESES}

At present the prosthetics designed for the upper limb are still in the stage of infancy. Hence a good reconstruction, even with a partial hand, is superior to a prosthetic hand.

Although great progress has been made in terms of design and control systems, all the current prostheses are myoelectric. Each movement relies on one voluntary muscle contraction, resulting in a slow stepwise motion as opposed to the fluid composite movement of the natural limb. A second issue is that the prostheses are insensate.

Considerable research is under way around the world to establish a neural interface between the nerve and prostheses. ${ }^{10}$ In the near future bionic replacements will become commonplace and part of the armamentarium for upper limb reconstruction.

\section{SUMMARY}

The successful treatment of mutilating injuries of the hand depends on correct assessment and execution of the surgical plan.

\section{REFERENCES}

1. Midgley RD, Entin MA. Management of mutilating injuries of the hand. Clin Plast Surg 1976;3(1):99-109.

2. Moran SL, Berger RA. Biomechanics and hand trauma: what you need. Hand Clin 2003;19(1): 17-31.

3. Peng YP, Lahiri A. Spare-part surgery. Semin Plast Surg 2013;27(4):190-7.

4. Soucacos PN. Indications and selection for digital amputation and replantation. J Hand Surg Br 2001; 26(6):572-81.

5. Buchler U. Traumatic soft-tissue defects of the extremities. Implications and treatment guidelines. Arch Orthop Trauma Surg 1990;109(6):321-9.

6. Sabapathy SR, Bajantri B. Indications, selection, and use of distant pedicled flap for upper limb reconstruction. Hand Clin 2014;30(2):185-99, vi.

7. Qing L, Wu P, Liang J, et al. Use of flow-through anterolateral thigh perforator flaps in reconstruction of complex extremity defects. J Reconstr Microsurg 2015;31(8):571-8.

8. Caroli A, Adani R, Castagnetti C, et al. Dorsalis pedis flap with vascularized extensor tendons for dorsal hand reconstruction. Plast Reconstr Surg 1993; 92(7):1326-30.

9. Katsaros J. Indications for free soft-tissue flap transfer to the upper limb and the role of alternative procedures. Hand Clin 1992;8(3):479-507.

10. Lahiri A, Delgado IM, Sheshadri S, et al. Self-organization of "fibro-axonal" composite tissue around unmodified metallic micro-electrodes can form a functioning interface with a peripheral nerve: a new direction for creating long-term neural interfaces. Muscle Nerve 2016;53(5):789-96. 